\title{
Brachial-ankle pulse wave velocity is an independent predictor of incident hypertension in Japanese normotensive male subjects
}

\author{
Hiroki Satoh · Yasuaki Saijo · Reiko Kishi • \\ Hiroyuki Tsutsui
}

Received: 8 May 2010/Accepted: 19 October 2010/Published online: 2 December 2010

(c) The Japanese Society for Hygiene 2010

\begin{abstract}
Objectives Cardiovascular morbidity and mortality are closely associated with hypertension, however, predictors of incident hypertension have not been fully established. We have conducted a study aimed at evaluating whether brachial-ankle pulse wave velocity (baPWV) is a predictor of incident hypertension.

Methods The relation between baPWV, a noninvasive index of aortic stiffness, and incident hypertension was evaluated in a cohort of 2,278 Japanese normotensive male subjects with a follow-up of 3 years.

Results Of the 2, 278 study participants, 151 (6.6\%) had incident hypertension during the follow-up. After adjustment for variables, including age, gender, body mass index (BMI), smoking habit, alcohol consumption, diabetes mellitus, hyperlipidemia, family history of hypertension, heart rate, systolic blood pressure, low-density lipoprotein cholesterol, triglyceride, and fasting plasma glucose, multiple logistic regression analysis revealed that baPWV was a significant and independent predictor of incident hypertension with an adjusted odds ratio 1.45 (95\% confidence
\end{abstract}

H. Satoh $(\bowtie) \cdot$ H. Tsutsui

Department of Cardiovascular Medicine, Hokkaido University

Graduate School of Medicine, Kita 15, Nishi 7,

Kita-ku, Sapporo, Hokkaido 060-8638, Japan

e-mail: h-satoh@imb.me-h.ne.jp

H. Satoh · R. Kishi

Department of Public Health Science, Hokkaido University

Graduate School of Medicine, Kita-ku, Sapporo,

Hokkaido, Japan

Y. Saijo

Department of Health Science, Asahikawa Medical College,

Asahikawa, Hokkaido, Japan interval $1.17-1.79, P<0.01)$. In addition, baPWV values $>1,380 \mathrm{~cm} / \mathrm{s}$ indicated a high risk for incident hypertension. Conclusions Among the Japanese normotensive male subjects participating in this study, BaPWV was a significant and independent predictor of incident hypertension. This result suggests that BaPWV could be a useful screening method to identify normotensive individuals who should be targeted for interventions aimed at preventing the incident hypertension.

Keywords Arterial stiffness - Hypertension - Pulse wave velocity - Cohort studies - Japanese male subjects

\section{Introduction}

The incidence and prevalence of hypertension increase with age. It has been well established that hypertension is associated with increased cardiovascular morbidity and mortality [1-5]. As such, it is of clinical importance to identify normotensive subjects who are at increased risk for developing hypertension and establish the conventional index that can be widely used in routine clinical practice. Previous studies have identified indices of central arterial stiffness assessed by ultrasound that could be used predict future hypertension in normotensive community population $[6,7]$. Najjar et al. demonstrated that carotid-femoral pulse wave velocity (PWV), a non-invasive marker of central arterial stiffness [8,9], could be an independent predictor of the longitudinal increase in blood pressure and incident hypertension in normotensive subjects [10].

Brachial-ankle (ba) PWV has been developed as a marker of arterial stiffness that can be measured using a noninvasive automatic device with relative technical simplicity. baPWV has been shown to be associated with 
intima-media thickness of the carotid artery and aortic PWV, a marker of central arterial stiffness [11, 12]. More importantly, baPWV is a significant and independent predictor of cardiovascular morbidity and mortality in patients on hemodialysis and with acute coronary syndrome [13, 14]. However, the relationship between baPWV and the development of hypertension has not been fully established, especially in Japanese normotensive male subjects.

The aim of the study reported here was to determine whether baPWV is an independent predictor of incident hypertension in Japanese normotensive subjects during a 3-year follow-up.

\section{Methods}

\section{Study subjects}

The study cohort comprised 2,656 Japanese male employees (age range 35-55 years) of a single local government agency who had an annual health checkup between April 2003 and March 2004. Of these, 257 were excluded for the following reasons: past history of coronary artery disease or stroke $(n=99)$, under treatment for hypertension $(n=114)$, low ankle/brachial index $<0.9(n=10)$, triglyceride values $>400 \mathrm{mg} / \mathrm{dL}(n=34)$. Ultimately, 121 $(4.6 \%)$ subjects were lost from observation during the follow-up period. Thus, a total of 2,278 subjects were successfully re-examined at the end of the 3-year follow-up.

This study was approved by the Institutional Ethical Board for epidemiological studies of Hokkaido University School of Medicine. Written informed consent was provided by all subjects.

\section{Baseline data measurements}

All subjects were asked to complete a self-administered questionnaire that includes questions on medical history, family history, smoking habit, alcohol consumption, and the frequency of exercise. The questionnaire was distributed to the subjects in advance of their annual check-up and was collected at the examination. Smoking habit and alcohol intake were determined from information collecting during interviews. Subjects who had never smoked and ex-smokers were classified as "nonsmokers". Drinkers were defined as those who consumed alcohol once per week or more. "Nonexercise" referred to the category of individuals who regularly exercised less than once per week. Body mass index (BMI) was calculated as body weight $(\mathrm{kg})$ divided by squared height (m). A blood sample was obtained from the antecubital vein in the morning after an overnight fast and serum was separated. After precipitation by heparin-manganese, total cholesterol and high-density lipoprotein
(HDL)-cholesterol were measured by the phosphotungstate method. Triglyceride was measured enzymatically. Glucose was enzymatically determined by the hexokinase method. Low-density lipoprotein (LDL)-cholesterol values were calculated using Friedwald formula [15]. BaPWV, ankle/ brachial index, and heart rate were measured with a volumeplethysmographic apparatus (model BP-203RPE II; Nihon Colin, Tokyo, Japan) $[11,16]$. The BaPWV was calculated by time-phase analysis between the right brachial and volume waveforms at both ankles. The ankle/brachial index is the ratio of ankle systolic blood pressure to brachial systolic blood pressure; right and left ankle/brachial indexes were measured simultaneously.

Blood pressure was measured by a trained nurse using a standard mercury sphygmomanometer, with the study subjects in the sitting position after at least a 5-min rest. Blood pressure measurements and information on pharmacological treatments of hypertension were assessed twice during the study in the same manner: at the beginning and the end of the 3-year follow-up.

\section{Incident hypertension}

Incident hypertension subjects were those normotensive male subjects who at the beginning of the follow-up had a systolic blood pressure $\geq 140 \mathrm{mmHg}$ and/or diastolic blood pressure $\geq 90 \mathrm{mmHg}$ at the end of the 3 -year follow-up were newly receiving anti-hypertensive drugs.

\section{Statistical analysis}

The clinical and biochemical data are expressed as the mean \pm standard deviation (SD), as the median value and interquartile range (IQR) for variables with a skewed distribution, and as the number and percentage. The differences in variables between two groups were examined by the Student unpaired $t$ test for approximately normal distributed variables, by the Wilcoxon rank-sum test for triglycerides, and by the $\chi^{2}$ test for the proportion of gender, alcohol consumption, smoking status, exercise habit, medical history of diabetes mellitus and hyperlipidemia, and family history of hypertension. The data were compared among the groups using analysis of variance (ANOVA), the KruskalWallis test, or the Cochran-Armitage test. The relationship between baPWV values and incident hypertension was investigated using multiple logistic regression analysis adjusted for baseline variables, such as age, alcohol consumption, smoking status, exercise habit, medical history of diabetes mellitus and hyperlipidemia, family history of hypertension, heart rate, BMI, LDL-cholesterol, triglyceride, HDL-cholesterol, fasting plasma glucose, and systolic blood pressure. The baPWV values were divided into quartiles with the following cut-off points: 1,204, 1,288, and 
$1,380 \mathrm{~cm} / \mathrm{s}$ for the second, third, and fourth quartiles, respectively. Multiple logistic regression analysis was examined by quartile levels of baPWV values adjusted for baseline variables, such as age, alcohol consumption, smoking status, exercise habit, medical history of diabetes mellitus and hyperlipidemia, family history of hypertension, heart rate, BMI, LDL-cholesterol, triglyceride, HDL-cholesterol, fasting plasma glucose, and systolic blood pressure.

A $P$ value $<0.05$ was considered to indicate statistical significance. All statistical analyses were performed using the SPSS ver. 11.0 software (SPSS, Chicago, IL).

\section{Results}

Of the 2,399 normotensive male subjects at baseline, 2,278 (95.0\%) were available for the 3-year follow-up assessment. There were 151 subjects who developed hypertension during the follow-up, with a cumulative incidence of $6.6 \%$. The baseline characteristics of the study subjects according to incident hypertension are presented in Table 1. Compared with normotensive subjects, subjects who developed hypertension during the follow-up significantly had more alcohol consumption, lower exercise habit, diabetes mellitus, hyperlipidemia, and higher values of baseline age, BMI, triglyceride, glucose, and baPWV. They also had significantly higher baseline systolic blood pressure and diastolic blood pressure, although these values were low the definite values of hypertension.

Odds ratios and the $95 \%$ confidence intervals (CI) of the relationship between baPWV and incident hypertension by multiple logistic regression analysis are presented in Table 2. In the age-adjusted model (Model 1), the OR of baPWV $( \pm 1 \mathrm{SD})(142 \mathrm{~cm} / \mathrm{s})$ increased by incident hypertension was 2.16 (95\% CI 1.80-2.60, $P<0.001)$. In the fully adjusted model (Model 3), this was 1.45 (95\% CI 1.17-1.79, $P<0.01)$.

Further assessment of the baseline characteristics of the study subjects stratified by the quartiles of baPWV resulted in values of $\leq 1,204,1,205-1,288,1,289-1,380$, and $\geq 1,381 \mathrm{~cm} / \mathrm{s}$ for quartiles $1-4$, respectively (Table 3 ). Compared to baPWV values in the lowest quartile, There was a trend for the prevalence of diabetes mellitus, hyperlipidemia, and family history of hypertension, age, BMI, heart rate, systolic and diastolic blood pressure values, and triglyceride and glucose levels to increase from the lowest quartile of baPWV to the highest quartile. The reverse trend was seen for prevalence of exercise.

Table 4 shows the results of the multiple logistic regression analysis evaluating the association between baPWV and incident hypertension as compared with that in the lowest quartile. The highest quartile level of baPWV $(>1,380 \mathrm{~cm} / \mathrm{s})$ could significantly and independently predict incident hypertension. The age-adjusted OR was 11.05

Table 1 Baseline characteristics of the study subjects in relation to the development of hypertension

\begin{tabular}{|c|c|c|c|c|}
\hline \multirow[t]{2}{*}{ Baseline characteristics } & \multirow[t]{2}{*}{ Total $(n=2,278)$} & \multicolumn{2}{|c|}{ Incident hypertension } & \multirow[t]{2}{*}{$P$ value } \\
\hline & & No $(n=2,127)$ & Yes $(n=151)$ & \\
\hline Age, years $(n)$ & $46 \pm 6$ & $46 \pm 6$ & $48 \pm 5$ & $<0.01$ \\
\hline Body mass index $\left(\mathrm{kg} / \mathrm{m}^{2}\right)$ & $23.7 \pm 2.8$ & $23.1 \pm 2.9$ & $25.4 \pm 3.3$ & $<0.001$ \\
\hline Smoking $(n, \%)$ & $1,164(51.1 \%)$ & $1,097(51.6 \%)$ & $67(44.4 \%)$ & 0.09 \\
\hline Alcohol $(n, \%)$ & $1,640(72.0 \%)$ & $1,519(71.4 \%)$ & $121(80.1 \%)$ & $<0.05$ \\
\hline Exercise $(\geq 1 /$ week $)(n, \%)$ & $1,035(45.4 \%)$ & $949(44.6 \%)$ & $86(57.0 \%)$ & $<0.01$ \\
\hline Diabetes mellitus $(n, \%)$ & $40(1.8 \%)$ & $31(1.5 \%)$ & $9(6.0 \%)$ & $<0.001$ \\
\hline Hyperlipidemia $(n, \%)$ & $88(3.9 \%)$ & $69(3.2 \%)$ & $19(12.6 \%)$ & $<0.001$ \\
\hline Family history of hypertension $(n, \%)$ & $317(13.9 \%)$ & $291(13.7 \%)$ & $26(17.2 \%)$ & 0.23 \\
\hline Heart rate (beats/min) & $61 \pm 10$ & $61 \pm 10$ & $62 \pm 10$ & 0.06 \\
\hline Systolic blood pressure (mmHg) & $117 \pm 9$ & $116 \pm 9$ & $127 \pm 7$ & $<0.001$ \\
\hline Diastolic blood pressure $(\mathrm{mmHg})$ & $74 \pm 7$ & $73 \pm 7$ & $82 \pm 5$ & $<0.001$ \\
\hline baPWV $(\mathrm{cm} / \mathrm{s})$ & $1,301 \pm 142$ & $1,293 \pm 137$ & $1,412 \pm 168$ & $<0.001$ \\
\hline LDL-cholesterol (mg/dL) & $127 \pm 31$ & $127 \pm 31$ & $126 \pm 32$ & 0.68 \\
\hline HDL-cholesterol (mg/dL) & $56 \pm 14$ & $56 \pm 14$ & $54 \pm 15$ & 0.09 \\
\hline Triglyceride $^{\mathrm{a}}(\mathrm{mg} / \mathrm{dL})$ & $104(73-150)$ & $103(73-149)$ & $115(86-169)$ & $<0.01$ \\
\hline Glucose (mg/dL) & $93 \pm 17$ & $93 \pm 16$ & $100 \pm 23$ & $<0.001$ \\
\hline
\end{tabular}

$b a P W V$ Brachial-ankle pulse wave velocity, $L D L$ low-density lipoprotein, $H D L$ high-density lipoprotein

Unless indicated otherwise, data are expressed as the mean \pm standard deviation (SD)

a Triglyceride is expressed as a median and interquartile range (IQR) due to its skewed distribution 
(95\% CI 5.24-23.29, $P<0.001, P$ for trend $<0.001)$ and the full adjusted OR was 2.70 (95\% CI 1.18-6.17, $P<0.05, P$ for trend $=0.01)$.

\section{Discussion}

The results reported here clearly show that baPWV was a significant and independent marker to predict incident hypertension among our study cohort of Japanese normotensive male subjects and that baPWV values $>1,380 \mathrm{~cm} / \mathrm{s}$

Table 2 Odds ratios and 95\% confidence interval of the relationship between brachial-ankle pulse wave velocity and incident hypertension by multiple logistic regression analysis

\begin{tabular}{llll}
\hline Model & Odds ratio & $95 \%$ CI & $P$ value \\
\hline Model 1 & 2.16 & $1.80-2.60$ & $<0.001$ \\
Model 2 & 1.98 & $1.69-2.31$ & $<0.001$ \\
Model 3 & 1.45 & $1.17-1.79$ & $<0.01$ \\
\hline
\end{tabular}

CI confidence interval

Odds ratio was indicated by a $1 \mathrm{SD}(142 \mathrm{~cm} / \mathrm{s})$ increase of baPWV values

Model 1 is adjusted for age; Model 2, model 1 plus adjustments for body mass index, smoking, alcohol, exercise, diabetes mellitus, hyperlipidemia, family history of hypertension, heart rate, low-density lipoprotein cholesterol, high-density lipoprotein cholesterol, triglyceride, and glucose; Model 3, model 2 plus adjustments for systolic blood pressure was a high risk factor for the development of hypertension during the 3-year follow-up.

The development of hypertension in normotensive subjects is associated with gender and advancing age [6]. Within the framework of the Framingham study, Vasan et al. [17] demonstrated that over a 4-year period elderly patients with high-normal and normal blood pressure were prone to develop incident hypertension. However, the prediction of future incident hypertension from blood pressure values alone have proved difficult [18]. Previous studies have found that the increased arterial stiffness could be useful markers to predict the development of hypertension [6, 7, 10, 19]. In the Atherosclerosis Risk in Communities (ARIC) study of normotensive subjects, Liao et al. [7] demonstrated that an increase in arterial stiffness of the left common carotid artery measured by high-resolution B-mode ultrasonography was associated with a $15 \%$ greater risk for the occurrence of hypertension, independent of conventional risk factors, including baseline blood pressure values. Based on results on normotensive subjects followed for 4 years, Dernellis et al. [20] reported that the arterial stiffness markers, such as aortic strain, destensibility, and the stiff index $(\beta)$, evaluated by M-mode echocardiography using a technique of polynominal regression analysis could be a predictor of incident hypertension after adjustment for conventional risk factors [6]. The findings in our study are in agreement with these previous studies. The significance of our study is that baPWV was found to be an independent predictor of future

Table 3 Baseline characteristics of the study subjects stratified by the quartiles of baPWV values

\begin{tabular}{|c|c|c|c|c|c|}
\hline Baseline characteristics & $\begin{array}{l}\text { Quartile } 1: \\
\leq 1,204 \mathrm{~cm} / \mathrm{s} \\
(n=573)\end{array}$ & $\begin{array}{l}\text { Quartile } 2: \\
1,205-1,288 \mathrm{~cm} / \mathrm{s} \\
(n=569)\end{array}$ & $\begin{array}{l}\text { Quartile 3: } \\
1,289-1,380 \mathrm{~cm} / \mathrm{s} \\
(n=568)\end{array}$ & $\begin{array}{l}\text { Quartile } 4: \\
\geq 1,381 \mathrm{~cm} / \mathrm{s} \\
(n=568)\end{array}$ & $\begin{array}{l}P \text { for } \\
\text { trend }\end{array}$ \\
\hline Age, years $(n)$ & $44 \pm 6$ & $45 \pm 6$ & $47 \pm 6$ & $48 \pm 6$ & $<0.001$ \\
\hline Body mass index $\left(\mathrm{kg} / \mathrm{m}^{2}\right)$ & $23.4 \pm 2.6$ & $23.5 \pm 2.7$ & $23.9 \pm 2.9$ & $23.8 \pm 2.8$ & $<0.01$ \\
\hline Smoking (yes) $(n, \%)$ & $291(50.7 \%)$ & $294(51.7 \%)$ & $281(49.5 \%)$ & $298(52.5 \%)$ & 0.77 \\
\hline Alcohol (yes) $(n, \%)$ & $404(70.5 \%)$ & $417(73.3 \%)$ & $392(69.0 \%)$ & $427(75.2 \%)$ & 0.09 \\
\hline Exercise $(\geq 1 /$ week $)(n, \%)$ & $288(50.2 \%)$ & $250(43.9 \%)$ & $266(46.8 \%)$ & $231(40.7 \%)$ & $<0.01$ \\
\hline Diabetes mellitus $(n, \%)$ & $1(0.2 \%)$ & $6(1.1 \%)$ & $10(1.8 \%)$ & $23(4.0 \%)$ & $<0.001$ \\
\hline Hyperlipidemia $(n, \%)$ & $15(2.6 \%)$ & $13(2.3 \%)$ & $25(4.4 \%)$ & $35(6.2 \%)$ & $<0.01$ \\
\hline Family history of hypertension $(n, \%)$ & $58(10.1 \%)$ & $87(15.3 \%)$ & $81(14.3 \%)$ & $91(16.0 \%)$ & $<0.05$ \\
\hline Heart rate (beats/min) & $58 \pm 8$ & $60 \pm 9$ & $61 \pm 10$ & $65 \pm 11$ & $<0.001$ \\
\hline Systolic blood pressure $(\mathrm{mmHg})$ & $110 \pm 8$ & $115 \pm 8$ & $118 \pm 9$ & $123 \pm 8$ & $<0.001$ \\
\hline Diastolic blood pressure $(\mathrm{mmHg})$ & $69 \pm 7$ & $73 \pm 7$ & $75 \pm 7$ & $79 \pm 6$ & $<0.001$ \\
\hline LDL-cholesterol (mg/dL) & $125 \pm 31$ & $125 \pm 31$ & $128 \pm 30$ & $128 \pm 33$ & 0.19 \\
\hline HDL-cholesterol (mg/dL) & $57 \pm 14$ & $56 \pm 14$ & $56 \pm 14$ & $56 \pm 14$ & 0.22 \\
\hline Triglyceride $^{\mathrm{a}}$ (mg/dL) & $90(66-130)$ & $101(70-150)$ & $108(76-151)$ & $120(84-167)$ & $<0.001$ \\
\hline Glucose (mg/dL) & $89 \pm 10$ & $92 \pm 13$ & $93 \pm 14$ & $99 \pm 25$ & $<0.001$ \\
\hline
\end{tabular}

Unless indicated otherwise, data are expressed as the mean \pm standard deviation (SD)

a Triglyceride is expressed as a median and interquartile range (IQR) due to its skewed distribution 
Table 4 Odds ratios and $95 \%$ CI for incident hypertension among quartiles of baPWV values
Models 1-3 are as described in footnote to Table 2

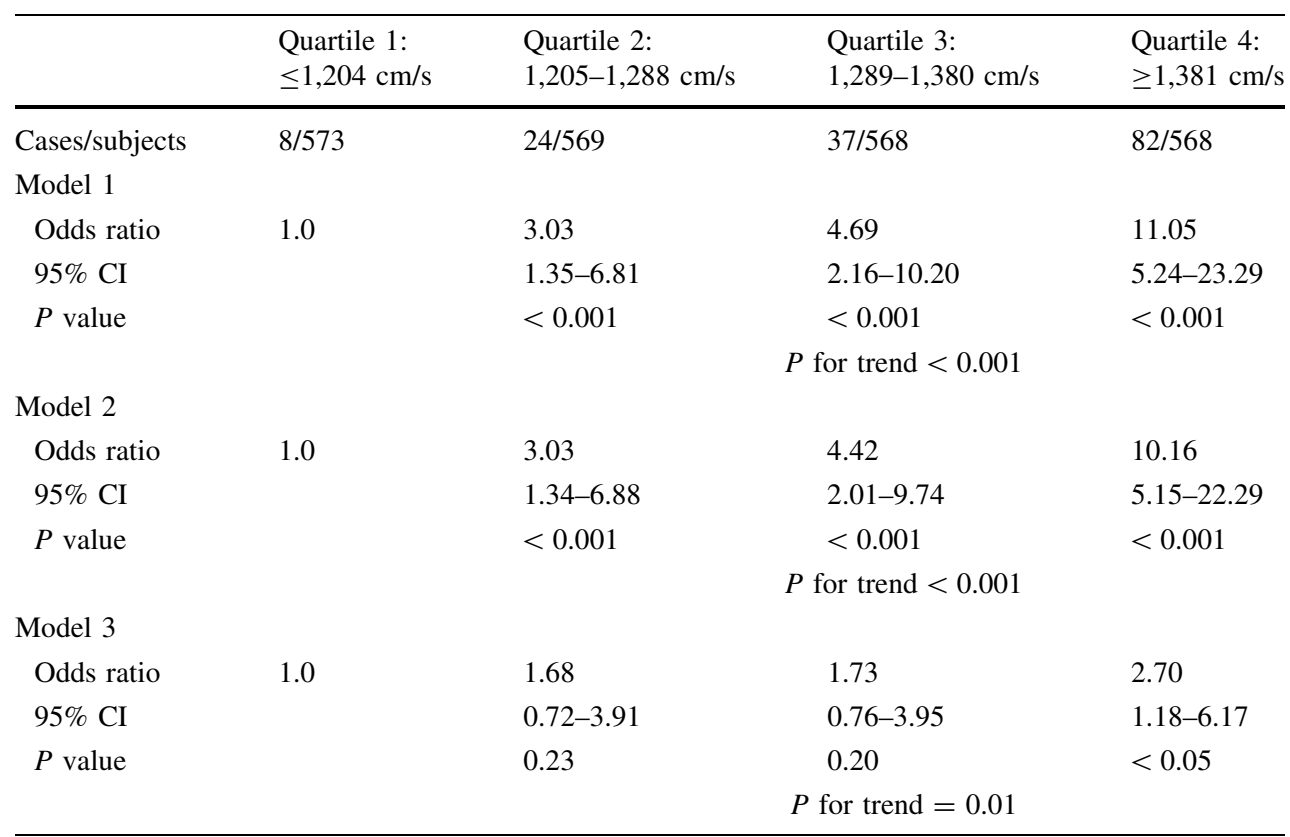

hypertension. It should be noted that unlike the echocardiographic methods used in previous studies, baPWV could become the most widely used conventional index of arterial stiffness.

PWV can be used as a predictor of cardiovascular events [21-28]. The carotid-femoral PWV measurement has been used as a conventional and common procedure [29]. baPWV measurements have recently been widely applied using a procedure in which pressure cuffs are wrapped around the brachial artery. In comparison with carotidfemoral PWV, baPWV can be widely applied during routine medical examinations because its measurement is simple and does not require specialized and highly technical equipment and expertise $[12,30]$. The measurements have been reported to be valid and reproducible and easier to perform than other noninvasive automatic procedures [11, 31, 32]. The baPWV values have been found to be correlated with intima-media thickness of the carotid artery and closely associated with aortic PWV, an index of central arterial stiffness $[11,12]$. In agreement with the results reported here, Yambe et al. [19] found that baPWV was predictive of progression to hypertension in subjects with high-normal blood pressure. However, these researchers had excluded subjects with dyslipidemia, diabetes mellitus, and fasting plasma glucose $>125 \mathrm{mg} / \mathrm{dL}$ and, consequently, the results may not be applicable to a normotensive general population. This is an important difference between our study and that of Yambe et al. [19].

There are a number of possible explanations of how high baPWV values could predict the incident hypertension. Aortic stiffness can be evaluated by the measurement of baPWV [14]. Stefanadis et al. [33] demonstrated that aortic stiffness was a cause of the premature return of reflected waves in late systole, resulting in increasing central aortic pressure; it was also closely associated with increased afterload. Thus, the elevation of afterload developed into hypertension. Wilkinson et al. [34] have suggested that nitric oxide plays an important role in the development of arterial stiffness as well as hypertension because nitric oxide released from the endothelium cells can alter vascular tone, cell growth and angiotensin receptors. Kameyama et al. [35] demonstrated that the inhibition of nitric oxide synthesis inhibition increased PWV values and, importantly, that their increase preceded the observed vascular structural changes in a rat model. These findings suggest that baPWV values increase as a result of decreases in nitric oxide synthesis and that the latter may be involved in the development of incident hypertension.

There are several limitations to our study that should be acknowledged. First, baPWV is an indirect marker of increased arterial stiffness or decreased arterial compliance, and we did not examine the structural changes of arterial wall using ultrasound technology in our study. Second, the constraints of our study did not allow us to determine the pathophysiological mechanisms linking increased arterial stiffness and the development of hypertension. Third, the identification of hypertension was performed twice (at the beginning of the study and at the end of the 3-year followup), and we were unable to examine detailed information on blood pressure, such as variations in blood pressure, during the follow-up period. Fourth, our study subjects were all from a single local government agency, and caution is recommended when applying these results to the general population. 
The major strength of our study is that we were able to demonstrate in a Japanese normotensive male study cohort that high baPWV values were closely associated with incident hypertension and that specific baPWV values were associated with a high risk of future incident hypertension. Many previous studies have demonstrated the relationship between incident hypertension and arterial stiffness based on baPWV values $[6,7,10,19]$, but these have not provided the appropriate baPWV values for incident hypertension.

In conclusion, Our results demonstrate that baPWV has the potential to be a significant and independent predictor for the development of hypertension in Japanese normotensive male subjects. They also suggest that baPWV could potentially be a useful and conventional screening method to identify normotensive subjects at a higher risk who could be a target for interventions aimed to prevent and delay the onset of hypertension.

Acknowledgments We thank Mrs Toshiko Kita, Dr Eiji Yoshioka, and Dr Tomonori Fukui for their excellent assistance for data collection.

\section{References}

1. Chobanian AV, Bakris GL, Black HR, Cushman WC, Green LA, Izzo JL Jr, et al. The Seventh Report of the Joint National Committee on prevention, detection, evaluation, and treatment of high blood pressure: the JNC 7 report. JAMA. 2003;289:2560-72.

2. Franco OH, Peeters A, Bonneux L, de Laet C. Blood pressure in adulthood and life expectancy with cardiovascular disease in men and women: life course analysis. Hypertension. 2005;46:280-6.

3. Kannel WB. Hypertension as a risk factor for cardiac eventsepidemiologic results of long-term studies. J Cardiovasc Pharmacol. 1993;21[Suppl 2]:S27-37.

4. Lloyd-Jones DM, Evans JC, Levy D. Hypertension in adults across the age spectrum: current outcomes and control in the community. JAMA. 2005;294:466-72.

5. Vasan RS, Larson MG, Leip EP, Evans JC, O'Donnell CJ, Kannel WB, Levy D. Impact of high-normal blood pressure on the risk of cardiovascular disease. N Engl J Med. 2001;345: 1291-7.

6. Dernellis J, Panaretou M. Aortic stiffness is an independent predictor of progression to hypertension in nonhypertensive subjects. Hypertension. 2005;45:426-31.

7. Liao D, Arnett DK, Tyroler HA, Riley WA, Chambless LE, Szklo M, Heiss G. Arterial stiffness and the development of hypertension. The ARIC study. Hypertension. 1999;34:201-6.

8. Asmar R, Benetos A, Topouchian J, Laurent P, Pannier B, Brisac AM, et al. Assessment of arterial distensibility by automatic pulse wave velocity measurement. Validation and clinical application studies. Hypertension. 1995;26:485-90.

9. Lehmann ED. Clinical value of aortic pulse-wave velocity measurement. Lancet. 1999;354:528-9.

10. Najjar SS, Scuteri A, Shetty V, Wright JG, Muller DC, Fleg JL, et al. Pulse wave velocity is an independent predictor of the longitudinal increase in systolic blood pressure and of incident hypertension in the Baltimore Longitudinal Study of Aging. J Am Coll Cardiol. 2008;51:1377-83.

11. Yamashina A, Tomiyama H, Takeda K, Tsuda H, Arai T, Hirose $\mathrm{K}$, et al. Validity, reproducibility, and clinical significance of noninvasive brachial-ankle pulse wave velocity measurement. Hypertens Res. 2002;25:359-64.

12. Yambe M, Tomiyama H, Hirayama Y, Gulniza Z, Takata Y, Koji $\mathrm{Y}$, et al. Arterial stiffening as a possible risk factor for both atherosclerosis and diastolic heart failure. Hypertens Res. 2004; 27:625-31.

13. Kitahara T, Ono K, Tsuchida A, Kawai H, Shinohara M, Ishii Y, et al. Impact of brachial-ankle pulse wave velocity and anklebrachial blood pressure index on mortality in hemodialysis patients. Am J Kidney Dis. 2005;46:688-96.

14. Tomiyama H, Koji Y, Yambe M, Shiina K, Motobe K, Yamada J, et al. Brachial-ankle pulse wave velocity is a simple and independent predictor of prognosis in patients with acute coronary syndrome. Circ J. 2005;69:815-22.

15. Friedewald WT, Levy RI, Fredrickson DS. Estimation of the concentration of low-density lipoprotein cholesterol in plasma, without use of the preparative ultracentrifuge. Clin Chem. 1972; 18:499-502.

16. Tomiyama H, Yamashina A, Arai T, Hirose K, Koji Y, Chikamori $\mathrm{T}$, et al. Influences of age and gender on results of noninvasive brachial-ankle pulse wave velocity measurement-a survey of 12517 subjects. Atherosclerosis. 2003;166:303-9.

17. Vasan RS, Larson MG, Leip EP, Kannel WB, Levy D. Assessment of frequency of progression to hypertension in non-hypertensive participants in the Framingham Heart Study: a cohort study. Lancet. 2001;358:1682-6.

18. Majahalme S, Turjanmaa V, Weder AB, Lu H, Tuomisto MT, Uusitalo A. Blood pressure level and variability in the prediction of blood pressure after 5-year follow-up. Hypertension. 1996; 28:725-31.

19. Yambe M, Tomiyama H, Yamada J, Koji Y, Motobe K, Shiina K, et al. Arterial stiffness and progression to hypertension in Japanese male subjects with high normal blood pressure. J Hypertens. 2007;25:87-93.

20. Stefanadis C, Dernellis J, Tsiamis E, Diamantopoulos L, Michaelides A, Toutouzas P. Assessment of aortic line of elasticity using polynomial regression analysis. Circulation. 2000;101:1819-25.

21. Cruickshank K, Riste L, Anderson SG, Wright JS, Dunn G, Gosling RG. Aortic pulse-wave velocity and its relationship to mortality in diabetes and glucose intolerance: an integrated index of vascular function? Circulation. 2002;106:2085-90.

22. Guerin AP, Blacher J, Pannier B, Marchais SJ, Safar ME, London GM. Impact of aortic stiffness attenuation on survival of patients in end-stage renal failure. Circulation. 2001;103:987-92.

23. Laurent S, Boutouyrie P, Asmar R, Gautier I, Laloux B, Guize L, et al. Aortic stiffness is an independent predictor of all-cause and cardiovascular mortality in hypertensive patients. Hypertension. 2001;37:1236-41.

24. Vaitkevicius PV, Fleg JL, Engel JH, O'Connor FC, Wright JG, Lakatta LE, et al. Effects of age and aerobic capacity on arterial stiffness in healthy adults. Circulation. 1993;88:1456-62.

25. Blacher J, Guerin AP, Pannier B, Marchais SJ, Safar ME, London GM. Impact of aortic stiffness on survival in end-stage renal disease. Circulation. 1999;99:2434-9.

26. Meaume S, Benetos A, Henry OF, Rudnichi A, Safar ME. Aortic pulse wave velocity predicts cardiovascular mortality in subjects $>70$ years of age. Arterioscler Thromb Vasc Biol. 2001;21: 2046-50.

27. Mattace-Raso FU, van der Cammen TJ, Hofman A, van Popele NM, Bos ML, Schalekamp MA, et al. Arterial stiffness and risk of coronary heart disease and stroke: the Rotterdam Study. Circulation. 2006;113:657-63.

28. Willum-Hansen T, Staessen JA, Torp-Pedersen C, Rasmussen S, Thijs L, Ibsen H, Jeppesen J. Prognostic value of aortic pulse wave velocity as index of arterial stiffness in the general population. Circulation. 2006;113:664-70. 
29. Davies JI, Struthers AD. Pulse wave analysis and pulse wave velocity: a critical review of their strengths and weaknesses. J Hypertens. 2003;21:463-72.

30. Munakata M, Ito N, Nunokawa T, Yoshinaga K. Utility of automated brachial ankle pulse wave velocity measurements in hypertensive patients. Am J Hypertens. 2003;16:653-7.

31. Munakata M, Nunokawa T, Yoshinaga K, Toyota T. Brachialankle pulse wave velocity is an independent risk factor for microalbuminuria in patients with essential hypertension-a Japanese trial on the prognostic implication of pulse wave velocity (J-TOPP). Hypertens Res. 2006;29:515-21.

32. Saijo Y, Yoshioka E, Fukui T, Kawaharada M, Kishi R. Metabolic syndrome, $\mathrm{C}$-reactive protein and increased arterial stiffness in Japanese subjects. Hypertens Res. 2006;29:589-96.
33. Stefanadis C, Dernellis J, Vlachopoulos C, Tsioufis C, Tsiamis E, Toutouzas $\mathrm{K}$, et al. Aortic function in arterial hypertension determined by pressure-diameter relation: effects of diltiazem. Circulation. 1997;96:1853-8.

34. Wilkinson IB, Franklin SS, Cockcroft JR. Nitric oxide and the regulation of large artery stiffness: from physiology to pharmacology. Hypertension. 2004;44:112-6.

35. Kameyama H, Takeda K, Kusaba T, Narumiya H, Tanda S, Kuwahara N, et al. Augmentation of pulse wave velocity precedes vascular structural changes of the aorta in rats treated with $N$ (omega)-nitro-L-arginine methyl ester. Hypertens Res. 2005;28: 439-45. 